\title{
Upper and Lower Bounds of Constrained Capacity in Diffusion-based Molecular Communication
}

\author{
Francesca Ratti ${ }^{\ddagger}$, Fardad Vakilipoor ${ }^{\ddagger}$, Maurizio Magarini ${ }^{\ddagger}$, and Hamdan Awan* \\ ${ }_{\ddagger}^{\ddagger}$ Dipartimento di Elettronica, Informazione e Bioingegneria \\ Politecnico di Milano, Milano, Italy \\ *Telecommunications Software and Systems Group (TSSG) \\ Waterford Institute of Technology, Ireland \\ Email: francesca.ratti@polimi.it, fardad.vakilipoor@mail.polimi.it, maurizio.magarini@polimi.it, hawan@tssg.org
}

\begin{abstract}
This paper investigates upper and lower bounds for the constrained capacity of a diffusive molecular communication (MC) system in the case where the information is associated with the concentration of molecules released by the transmitter. The evaluation of channel capacity for the diffusive channel is an open problem in the context of MC. Here, two simple bounds of the constrained capacity are derived for a given number of input concentration levels. Numerical results are reported for binary and quadruple concentration-shift keying considering the Poisson and Gaussian distributions, which are two common approximations used to describe the statistics of the received signal. We show that for both the two statistical channel models the resulting bounds are tight and, therefore, this means that, at least for low modulation orders, it is not necessary to resort to numerical techniques or complicated analytical expressions to guess the capacity of the diffusive MC channel.
\end{abstract}

\section{INTRODUCTION}

In recent years researchers in the fields of biology and communication have become increasingly interested in studying molecular communication (MC) [1]. An important research problem in this type of communication is that of finding an optimal way to transfer information from a source (transmitter) to a destination (receiver) cell. As a consequence, there has been a growing interest in the calculation of channel capacity and of information transfer rate in MC networks [2], [3].

In this work we focus on MC systems where molecules released at the transmitting cell propagate to the receiving cell via diffusion. A complete characterization of the diffusive MC channel can be found in [4]. As far as the statistical characterization is concerned, it is shown in [4] that both the Poisson and the Gaussian distribution are suitable models to analyze the performance of the MC channel. Both these two channels have been very well studied and many tighter bounds, analytical expressions or numerical methods can be found in the literature to support the evaluation of channel capacity.

With focus on analytical derivations, the authors of [5] provide a closed-form expression for the information capacity of an MC system with a noisy channel. Similarly, in [6] the authors analyze the capacity of the channel considering both the diffusion-based channel and the ligand-based receiver. Another work in [7] presents the analysis of the channel capacity in diffusive MC by considering intersymbol interference (ISI) from all the previous time slots and the channel transmission probability in each of them. The results in their work show that the channel capacity can be maximized with an optimal lower bit error probability by controlling the channel transmission probability in each time slot. Similarly, in [8] an upper bound on the capacity is calculated for a Poisson channel with a maximum transmission constraint in the low signal-to-noise ratio regime. In [9] a different approach is taken where the authors use enzymatic reaction cycles to improve the upper bound of mutual information for a diffusion-based communication system. Also, the capacity of these channels can be evaluated numerically by using Blahut-Arimoto algorithm or its variants [10].

In this paper we aim to address the evaluation of the discrepancies in the channel capacity when the Poisson and the Gaussian models are used for the statistical characterization of the received signal. As a main contribution, we evaluate upper and lower bounds for the constrained capacity of an MC system with a single transmitting and a single receiving cell for discrete-time memoryless Poisson and Gaussian channel models. We show that for the two considered modulations both the two bounds are quite tight, especially at high signalto-noise (SNR), and no significant differences are observed between the two approximations. This means that both them can be considered in the definition of the bounds with respect to which to compare the constrained capacity evaluated by means of closed form expressions or numerical techniques.

The remainder of this paper is organized as follows. First in Sec. II, we explain the system model including transmitter, channel, and receiver. Next in Sec. III we present the assumptions for both the cases of Poisson and Gaussian statistical description. In Sec. IV we derive the upper and the lower bounds on the capacity and present the mathematical expressions. Section V presents numerical results. Finally, in Sec. VI we conclude the paper.

\section{SySTEM MODEL}

The main contribution of this paper is to present a method to define bounds against which to compare the results achieved with in-silico simulations of engineered cell-to-cell communication system reactions. In this work the system is composed of one transmitting cell and one receiving cell that exchange information through molecules propagating in a diffusive 
channel. We derive the upper and lower bounds on the channel capacity for two different scenarios: (i) when the received signal is modelled by means of a Poisson distribution and (ii) when the noise that affects the received signal is assumed to be Gaussian distributed. We note that the upper bound calculated in this paper differs from the work in [8]. This is because in our work instead of using the symmetrized Kullback-Leibler divergence, we implement the definition of this divergence that compares it to the relative entropy between two variables [11]. We present a comparison between the upper and lower bounds of capacity for both these cases. We learn that the increase in the concentration of the system input results in an exponential increase of both the upper and the lower bounds. Furthermore, we realize that the Gaussian noise leads to an overestimation of the upper bound at lower values of the input concentration.

With reference to the considered scenario, we assume that the distance between the centers of the transmitting and the receiving cells is $d=1 \mu \mathrm{m}$. The transmission of molecules works by inducing a molecule concentration gradient between transmitter and receiver. We assume that the transmitter releases a certain amount of specific signaling molecules in the diffusive medium. As a result the molecules diffuse in the environment from the transmitter towards the location of the receiver. Although the receiving cell has specific receptors on its surface to capture these particular signaling molecules, in our model we consider a fully transparent receiver where the time-varying concentration of the received molecules carries the message to be decoded by the receiver. When the molecules diffuse from one end to another they are affected by the noise in the environment. This noise has a significant impact on the communication performance of the system. In this work we specifically aim to compute the upper and lower bounds of the constrained capacity for the two above mentioned types of approximations used to describe the statistics of the received signal.

We assume an inter-cellular environment where the signaling molecules propagate from the transmitter cell to the receiver cell by following a random walk Brownian motion [9], [12]. On reaching the receiver the number of molecules inside the cell is counted (transparent assumption). A radius $r_{T X / R X}=0.15 \mu \mathrm{m}$ is considered for the spherical transmitting and receiving cells. The respective volume can be calculated by using the standard formula: $V_{T X / R X}=\frac{4}{3} \pi r_{T X / R X}^{3}$.

\section{Statistical Approximations}

In this section we explain how we model the impulse response and the channel noise for two different cases. The observation of molecules at the receiver can be described as a binomial distribution since they are either observed by the receive or not [4]. We can approximate the binomial distribution with Poisson and Gaussian distributions under some specific circumstances as will be detailed in following subsections. For both the cases the channel is assumed to be discrete and memoryless. We assume that the input signal is constant with time, as we generate random Poisson and Gaussian samples for the received signal. In this paper we use a BCSK or QCSK modulation to transmit the information on the channel. CSK is the most simple modulation technique where the amplitude of the signal is considered to be the number of the received molecules in a time slot. We do not consider ISI. Next the two assumptions are presented.

\section{A. Poisson assumption}

For this case we consider that the input concentration values range from $10^{4} \mathrm{nM}$ to $3 \times 10^{5} \mathrm{nM}$ with step size of $10^{4} \mathrm{nM}$. Note that for the purpose of explanation we consider QCSK in this section. For each simulation, we take one value of concentration and create the $\lambda$ value of the four Poisson distributions of the QCSK by multiplying it by 1, 2, 3, and 4 . Next for each result we generate the samples of the distribution by using the built in Poisson probability density function (PDF) that is available MATLAB. In this way, we can obtain the samples for a Poisson distribution from the following formula:

$$
y=f(x \mid \lambda)=\frac{\lambda^{x}}{x !} \exp (-\lambda),
$$

where $\lambda$ that is the event rate of the distribution. For a transparent receiver, the impulse response can be written as [4]

$$
h(d, t)=\frac{V_{R X}}{(4 \pi D t)^{3 / 2}} \exp \left(-\frac{d^{2}}{4 D t}\right),
$$

where $D$ is the diffusion coefficient, $V_{R X}$ is the volume of the cell, and $d$ is the distance between the center of the transmitter and receiver cells as shown in Figure 1. For $N_{T X}$ molecules emitted by the transmitter at time $\tau=0 \mathrm{~s}$, the expected number of molecules observed at the receiving end at time $t$ is given by [4]

$$
\bar{y}(d, t)=N_{T X} h(d, t) .
$$

The arrival of molecules in MC via diffusion is a counting process, which exhibits a binomial distribution [13]. For large number of trials and small mean of the binomial random variable, a binomial distribution can be well approximated by a Poisson distribution with the same mean $\bar{y}(t)$ :

$$
y(d, t) \simeq \mathcal{P}\left(N_{T X} h(d, t)\right),
$$

where $\mathcal{P}(\lambda)$ represents a Poisson distribution with parameter $\lambda$. In case of QCSK, where four different concentrations of molecules are released, i.e. $N_{T X ; 1}, N_{T X ; 2}, N_{T X ; 3}$, and $N_{T X ; 4}$, are given by: $x_{i}(t)=N_{T X ; i} h(t)$

These equations provide the expected values of the observed molecules. We note that they do not depend on $d$ since we assume the transmitter and the receiver cells are at a fixed distance. In this way, we have:

$$
N_{i}=N_{T X ; i} V_{R X}
$$

Next, we consider the sampling at the maximum of the impulse response

$$
t_{\max }=\frac{d^{2}}{6 D}
$$




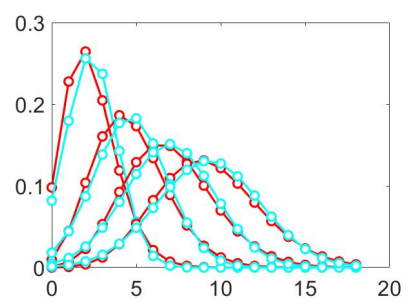

(a)

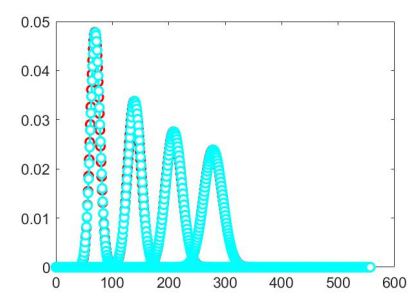

(b)
Fig. 1: (a) Comparison between the Poisson (red) and the Gaussian (light blue) distribution for a QCSK constellation and an input concentration of $10^{4} \mathrm{nM}$. (b) Comparison between the Poisson (red) and the Gaussian (light blue) distribution for a QCSK constellation and an input concentration of $3 \times 10^{5} \mathrm{nM}$.

where the maximum number of observed molecules is

$$
\begin{aligned}
x_{i} & =x_{i}\left(t_{\max }\right)=N_{T X ; i} h\left(t_{\max }\right) \\
& =N_{T X ; i} \frac{V_{R X}}{d^{3}}\left(\frac{3}{2 \pi e}\right)^{3 / 2}=\frac{N_{i}}{d^{3}}\left(\frac{3}{2 \pi e}\right)^{3 / 2} .
\end{aligned}
$$

This result is obtained by substituting (6) in (3) and (5). From [14] we can obtain the channel transition probability as

$$
P_{Y \mid X}\left(y \mid x_{i}\right)=\operatorname{Pr}\left[Y=y \mid X=x_{i}\right]=e^{-x_{i}} \frac{x_{i}^{y}}{y !}, y \in \mathcal{Z}^{+}, x_{i} \geq 0 .
$$

In general, assuming $K$ equiprobable concentration levels at the input, the distribution of the output is given by

$$
P_{Y}(y)=\frac{1}{K} \sum_{i=1}^{C} P_{Y \mid X}\left(y \mid x_{i}\right) .
$$

This means that for QCSK we have

$$
\begin{aligned}
& P_{Y}(y)= \\
& =\frac{1}{4} P_{Y \mid X}\left(y \mid x_{1}\right)+\frac{1}{4} P_{Y \mid X}\left(y \mid x_{2}\right)+\frac{1}{4} P_{Y \mid X}\left(y \mid x_{3}\right)+\frac{1}{4} P_{Y \mid X}\left(y \mid x_{4}\right) .
\end{aligned}
$$

\section{B. Gaussian assumption}

In this subsection, we describe the generation of the random Gaussian noise samples that give an approximation of the random changes around the mean value of the Poisson distributed received signal. This allows us to compute the impact of the Gaussian noise on the capacity of the system. We can further compare this approximation with that of the Poisson distribution for the received signal. We consider a Gaussian distribution with both the mean and the variance equal to $\lambda$, i.e. the parameter of the Poisson distribution defined in Sec. III-A. The equation that characterizes the Gaussian distribution is given by

$$
y=f(x \mid \mu, \sigma)=\frac{1}{\sigma \sqrt{2 \pi}} \exp \left(\frac{-(x-\mu)^{2}}{2 \sigma^{2}}\right),
$$

where $\mu$ represents the mean value and $\sigma$ the variance. As we obtain the received signal with Gaussian noise added, we proceed similarly to what done in Sec. III-A to calculate the channel transition probability and the PDF of the output using (8) and (9).

As shown in Figs. 1a and 1b, the Gaussian approximation of noise is almost equivalent to the Poisson approximation for high values of input concentration. On the other hand, the figures also show that the curves of the Poisson and the Gaussian approximations are not completely overlapping for low values of input concentration. This suggests that the capacity bounds calculation for Gaussian noise approximation will be less accurate as compared to the case of Poisson approximation for the received signal.

\section{CALCULATION OF THE BOUNDS ON THE CAPACITY}

In this section we calculate the upper and the lower bound on the capacity of an MC system for the two different approximations described in Sec. II. In order to achieve this, we need to derive the channel transition probability and the PDF of the output signal.

\section{A. The lower bound}

The expression for the capacity of a discrete memoryless channel with $P_{Y \mid X}(y \mid x)$ over the finite input and output, i.e. $\mathcal{X}$ and $\mathcal{Y}$, respectively, is given by [15]

$$
C=\max _{P_{X} \in \mathcal{P}(\mathcal{X})} I\left(P_{X} ; P_{Y \mid X}\right),
$$

where $\mathcal{P}(\mathcal{X})$ denotes the set of all probability measures on $\mathcal{X} . I\left(P_{X} ; P_{Y \mid X}\right)$ represents the mutual information when the input is distributed according to $P_{X}$. The mutual information is written as

$$
I\left(P_{X} ; P_{Y \mid X}\right)=\sum_{x, y} P_{X}(x) P_{Y \mid X}(y \mid x) \log _{2} \frac{P_{Y \mid X}(y \mid x)}{\left(P_{X} P_{Y \mid X}\right)(y)},
$$

where $\left(P_{X} P_{Y \mid X}\right)$ represents the output distribution corresponding to the input $P_{X}$, i.e.,

$$
\left(P_{X} P_{Y \mid X}\right)(y)=\sum_{x^{\prime}} P_{X}\left(x^{\prime}\right) P_{Y \mid X}\left(y \mid x^{\prime}\right), \quad y \in \mathcal{Y} .
$$

Although the optimization over input distribution complicates the exact computation of $C$, (12) leads to a very natural lower bound on $C$, since any input distribution $P_{X} \in \mathcal{P}(\mathcal{X})$ leads to the inequality

$$
C \geq I\left(P_{X} ; P_{Y \mid X}\right) .
$$

It is well known that for a continuous variable over a channel, the mutual information can be expanded in terms of differential entropy as

$$
I\left(P_{X} ; P_{Y \mid X}\right)=h(Y)-h(Y \mid X) .
$$

Since the channel is discrete, the values $h(Y)$ and $h(Y \mid X)$ can be obtained by using:

$$
h(Y)=-\sum_{i=1}^{n} P_{Y}\left(y_{i}\right) \log _{2}\left(P_{Y}\left(y_{i}\right)\right)
$$


where $P_{Y}\left(y_{i}\right)$ is calculated by using (9), and

$$
h(Y \mid X)=-\sum_{j=1}^{m} P_{X}\left(x_{j}\right) \sum_{i=1}^{n} P_{Y \mid X}\left(y_{i} \mid x_{j}\right) \log _{2}\left(P_{Y \mid X}\left(y_{i} \mid x_{j}\right)\right) .
$$

In (18), $P_{X}\left(x_{j}\right)$ represents the probability of the input distribution. Since all the possible input concentrations are considered equiprobable, $P_{X}\left(x_{j}\right)$ will be constant and it is calculated by dividing (1) for the number of possible input concentrations. The channel transition probability $P_{Y \mid X}\left(y_{i} \mid x_{j}\right)$ is then obtained from (8).

\section{B. The upper bound}

In this subsection we calculate the upper bound of the capacity. The mutual information of the system can be calculated as a Kullback-Leibler divergence of the product of the marginal distributions, $P_{X}(x)$ and $P_{Y}(y)$, from the joint distribution of the two random variables $X$ and $Y P_{X Y}(x, y)$ as [16]

$$
I(X ; Y)=D_{K L}\left(P_{X Y}(x, y) \| P_{X}(x) P_{Y}(y)\right) .
$$

The Kullback-Leibler divergence has the same equation as that of the relative entropy and is given as

$D_{K L}\left(P_{Y \mid X}(y \mid x) \| P_{Y}(y)\right)=\sum_{y \in \mathcal{Y}} P_{Y \mid X}(y \mid x) \log _{2} \frac{P_{Y \mid X}(y \mid x)}{P_{Y}(y)}$.

This means that we can rewrite (19) as

$$
I(X ; Y)=\sum_{x \in \mathcal{X}} P_{x}(x) D_{K L}\left(P_{Y \mid X}(y \mid x) \| P_{Y}(y)\right) .
$$

Therefore, a dual expression for the channel capacity similar to [15] is given by

$$
C=\min _{P_{Y} \in \mathcal{P}(\mathcal{Y})} \max _{x \in \mathcal{X}} D_{K L}\left(P_{Y \mid X}(y \mid x) \| P_{Y}(y)\right) .
$$

Every choice of a distribution $P_{Y}(y)$ on the output $\mathcal{Y}$ leads to an upper bound on channel capacity [15] as follows

$$
C \leq \max _{x \in \mathcal{X}} D_{K L}\left(P_{Y \mid X}(y \mid x) \| P_{Y}(y)\right) .
$$

To solve (23), we use the identity in [17]:

$$
\begin{aligned}
& \sum_{x \in \mathcal{X}} P_{X}(x) D_{K L}\left(P_{Y \mid X}(y \mid x) \| P_{Y}(y)\right)= \\
& \quad I\left(P_{X Y} ; P_{Y}\right)+D_{K L}\left(\left(P_{X} P_{Y \mid X}\right)(y) \| P_{Y}(y)\right)
\end{aligned}
$$

and since the relative entropy cannot be negative, we obtain the following relation:

$$
I\left(P_{X} ; P_{Y \mid X}\right) \leq \sum_{x \in \mathcal{X}} P_{X}(x) D_{K L}\left(P_{Y \mid X}(y \mid x) \| P_{Y}(y)\right),
$$

where $P_{Y} \in \mathcal{P}(\mathcal{Y})$ in (25) implies (23).

\section{Numerical Results}

In this section we first present the parameters selected for the numerical analysis and then we follow this up with a results and discussion section.

\section{A. Parameters}

The parameters used to characterize the MC system model are similar both for the case where we consider the Poisson approximation and for that when we consider Gaussian distribution. As already said in Sec. II, the radius of both the transmitter cell and the receiver cell is $r_{T X / R X}=0.15 \mu \mathrm{m}$. This value of radius is typical for a prokaryotic cell. The distance $d$ between the center of the transmitter and the receiver cell is equal to $1 \mu \mathrm{m}$. The input information is encoded in either two or four levels of concentration. To be specific we use both BCSK and QCSK modulation in this paper. The input concentration $C$ spans on a range of $10^{4} \mathrm{nM}$ to $3 \times 10^{5} \mathrm{nM}$ with step size $10^{4} \mathrm{nM}$. All of these values are equi-probable and are modulated by the two or four levels of the concentration. For the Poisson distribution presented in (4), we model the $\lambda$ parameter for each level corresponding to each possible concentration value by using:

$$
\lambda_{i j}=j \cdot x_{i}\left(t_{\max }\right)
$$

where the subscript $j$ varies from 1 and 4 depending on the level of the concentration that is being considered. The value of $x_{i}\left(t_{\max }\right)$ is calculated by using (7). The term $N_{i}$ in (7) is obtained by the multiplication of the volume of the receiver cell $V_{R X}$ and $C_{i}$, i.e. the current value of concentration that is input to the system. We evaluate the Poisson distribution in all the integer values between 0 and $\left[2 \cdot\left(4 x_{i}\left(t_{\max }\right)\right)\right]$, where 4 represents the number of levels in the constellation. In this way, the channel transition probability $P_{Y \mid X}(y \mid x)$ for each possible input concentration $i$ and for each level of the constellation $j$ is approximated by the Poisson distribution $\mathcal{P}\left(\lambda_{i j}\right)$. The PDF of the output is given by

$$
P_{Y}(y)=\frac{1}{K} \sum_{j=1}^{4} P_{Y \mid X}(y \mid x) .
$$

Similarly, for the case when the noise is approximated by a Gaussian distribution, all the values of the parameters are the same as those described above. We approximate the channel transition probability with a Gaussian distribution that has a mean value $\mu_{i j}$ equal to $\lambda_{i j}$, so that it can be calculated by using (26). Whereas the standard deviation $\sigma_{i j}$ is equal to the square root of $\lambda_{i j}$. For both these cases using the channel transition probability and the pdf of the output we calculate the upper and lower bounds of capacity by using the expressions in Sec. 4. In the next subsection we present and discuss these bound results for the channel capacity of the diffusive MC channel.

\section{B. Results}

In this subsection we present the results for the upper and lower bounds of the capacity for both Poisson and Gaussian approximations. Figure 2 presents the upper and the lower bound on the capacity for the Poisson distribution. In this figure we consider both the BCSK and QCSK case. As shown in the Fig. 2 both the upper and the lower bound tend to increase as the input concentration increases. The gap between 


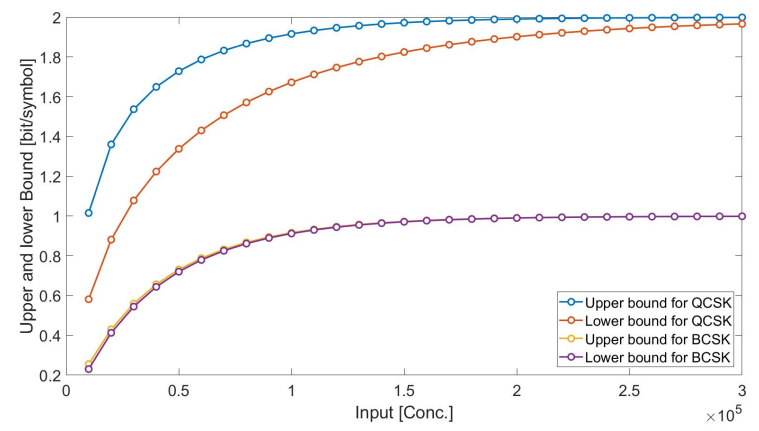

Fig. 2: Upper and lower bound on the capacity for BCSK and QCSK with Poisson channel model.

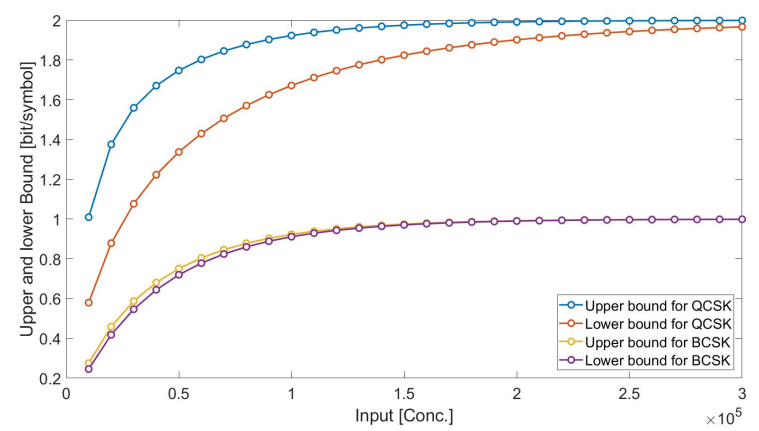

Fig. 3: Upper and lower bound on the capacity for BCSK and QCSK with Gaussian channel model.

both the bounds becomes narrower as the input concentration increases further. Next in Fig. 3 we present the upper and the lower bounds of capacity when the noise is approximated as a Gaussian distribution for both BCSK and QCSK case. Similar to the results shown in Fig. 2, both the upper and the lower bounds increase non-linearly with the increase in the input concentration of molecules. By comparing the results in Figs. 2 and 3 we realize that the Gaussian noise assumption leads to an overestimation of the upper bound for low concentration of the input. On the other hand, the results are very similar for a high concentration of the input. This is because for a low concentration of the input the Gaussian shape does not give a good approximation of the Poisson one, as already noticed in Sec. III-B. For both Figs. 2 and 3 we also learn that the upper and lower bounds are much closer for the BCSK case as compared to the QCSK case.

\section{CONCLUSIONS}

In this paper we have presented the analytical bounds on the capacity of an diffusive MC channel that give a reference against which it is possible to compare results of in-silico simulations or of experiments. We derived the upper and lower bounds of capacity for two scenarios: when the received signal is approximated by a Poisson distribution and when it is approximated by a Gaussian distribution. The results show that both the upper and the lower bounds tend to increase with the increase in input concentration. We further observed that for the lower values of input concentrations the Gaussian approximation leads to an overestimation of the upper bound as compared to the Poisson approximation. We further learned that for the BCSK case the bounds are much closer as compared to the QCSK case.

Future extensions of this work will focus on considering the effect of ISI in the evaluation of the constrained capacity for the waveform channel where continuous-time signaling is considered.

\section{REFERENCES}

[1] Ian F Akyildiz, Fernando Brunetti, and Cristina Blázquez. Nanonetworks: A new communication paradigm. Computer Networks, 52(12):2260-2279, 2008.

[2] Tadashi Nakano, Yutaka Okaie, and Jian-Qin Liu. Channel model and capacity analysis of molecular communication with brownian motion. IEEE communications letters, 16(6):797-800, 2012.

[3] Baris Atakan and Ozgur B Akan. On channel capacity and error compensation in molecular communication. In Transactions on computational systems biology $X$, pages 59-80. Springer, 2008.

[4] Vahid Jamali, Arman Ahmadzadeh, Wayan Wicke, Adam Noel, and Robert Schober. Channel modeling for diffusive molecular communication-a tutorial review. Proceedings of the IEEE, 107(7):1256-1301, 2019.

[5] Massimiliano Pierobon and Ian F Akyildiz. Capacity of a diffusion-based molecular communication system with channel memory and molecular noise. IEEE Transactions on Information Theory, 59(2):942-954, 2013.

[6] Qiang Liu and Kun Yang. Channel capacity analysis of a diffusion-based molecular communication system with ligand receptors. International Journal of Communication Systems, 28(8):1508-1520, 2015.

[7] Zhen Cheng, Yihua Zhu, Kaikai Chi, Yanjun Li, and Ming Xia. Capacity analysis for diffusive molecular communication with isi channel. Nano communication networks, 13:43-50, 2017.

[8] Gholamali Aminian, Hamidreza Arjmandi, Amin Gohari, Masoumeh Nasiri-Kenari, and Urbashi Mitra. Capacity of diffusion-based molecular communication networks over lti-poisson channels. IEEE Transactions on Molecular, Biological and Multi-Scale Communications, 1(2):188201, 2015.

[9] Hamdan Awan and Chun Tung Chou. Improving the capacity of molecular communication using enzymatic reaction cycles. IEEE transactions on nanobioscience, 16(8):744-754, 2017.

[10] Richard D Wesel, Emily E Wesel, Lieven Vandenberghe, Christos Komninakis, and Muriel Medard. Efficient binomial channel capacity computation with an application to molecular communication. In 2018 Information Theory and Applications Workshop (ITA), pages 1-5. IEEE, 2018.

[11] Thomas M Cover and Joy A Thomas. Elements of information theory. John Wiley \& Sons, 2012.

[12] Vahid Jamali, Arman Ahmadzadeh, Christophe Jardin, Heinrich Sticht, and Robert Schober. Channel estimation for diffusive molecular communications. IEEE Transactions on Communications, 64(10):4238-4252, 2016.

[13] H Birkan Yilmaz, Chan-Byoung Chae, Burcu Tepekule, and Ali E Pusane. Arrival modeling and error analysis for molecular communication via diffusion with drift. In Proceedings of the Second Annual International Conference on Nanoscale Computing and Communication, page 26. ACM, 2015.

[14] Amos Lapidoth and Stefan M Moser. The asymptotic capacity of the discrete-time poisson channel. Proceedings Winter School on Coding and Information Theory, Monte Verita, Ascona, Switzerland, 2003.

[15] Amos Lapidoth and Stefan M Moser. Capacity bounds via duality with applications to multiple-antenna systems on flat-fading channels. IEEE Transactions on Information Theory, 49(10):2426-2467, 2003.

[16] Imre Csiszar and János Körner. Information theory: coding theorems for discrete memoryless systems. Cambridge University Press, 2011.

[17] F Tops. An information theoretical identity and a problem involving capacity. Studia Scientiarum Mathematicarum Hungarica, 2:291-292, 1967. 